(n)

trobertivier Journal of Nonlinear Mathematical Physics

\title{
Inverse Spectral Problem and Peakons of an Integrable Two- component Camassa-Holm System
}

Fengfeng Dong, Lingjun Zhou

To cite this article: Fengfeng Dong, Lingjun Zhou (2018) Inverse Spectral Problem and Peakons of an Integrable Two-component Camassa-Holm System, Journal of Nonlinear Mathematical Physics 25:2, 290-308, DOI:

https://doi.org/10.1080/14029251.2018.1452674

To link to this article: https://doi.org/10.1080/14029251.2018.1452674

Published online: 04 January 2021 


\title{
Inverse Spectral Problem and Peakons of an Integrable Two-component Camassa-Holm System
}

\author{
Fengfeng Dong \\ School of Mathematical Sciences, Tongji University \\ Shanghai 200092, P.R. China \\ 1433494@tongji.edu.cn \\ Lingjun Zhou \\ School of Mathematical Sciences, Tongji University \\ Shanghai 200092, P.R. China \\ zhoulj@tongji.edu.cn
}

Received 19 November 2017

Accepted 17 December 2017

\begin{abstract}
In this paper, we are concerned with the explicit construction of peakon solutions of the integrable twocomponent system with cubic non-linearity. We establish the spectral and inverse spectral problem associated to the Lax pairs of the system. The inverse problem is solved by the classical results of Stieltjes continued fractions, which also contributes a lot to the spectral problem. The explicit formulas are obtained from solutions of the inverse problem. The positivity of the spectral measures is implied by J. Moser's work on the Jacobi spectral problem.
\end{abstract}

Keywords: peakon; two-component Camassa-Holm system; continued fractions; inverse spectral approach.

2000 Mathematics Subject Classification: 35Q51, 37K15, 34A55

\section{Introduction}

In this paper, we consider the following two-component system

$$
\left\{\begin{array}{l}
m_{t}=\frac{1}{2}\left[m\left(u v-u_{x} v_{x}\right)\right]_{x}-\frac{1}{2} m\left(u v_{x}-u_{x} v\right), \\
n_{t}=\frac{1}{2}\left[n\left(u v-u_{x} v_{x}\right)\right]_{x}+\frac{1}{2} n\left(u v_{x}-u_{x} v\right), \\
m=u-u_{x x}, \quad n=v-v_{x x} .
\end{array}\right.
$$

The completely integrable system (1.1) is proposed by Xia and Qiao in [45], it admits biHamiltonian structure and infinity many conservation laws and also integrable in the sense of geometry for describing pseudo-spherical surfaces. The blow-up scenario, persistence properties and unique continuation have been discussed in $[23,46]$.

When $v=-2,(1.1)$ is reduced to the Camassa-Holm $(\mathrm{CH})$ equation

$$
m_{t}+m_{x} u+2 m u_{x}=0, \quad m=u-u_{x x},
$$

which models the unidirectional propagation of shallow water waves over a flat bottom $[5,6,14,26]$, and can also be derived as the tri-Hamiltonian duality of the Korteweg-de Vries (KdV) equation [36]. Compared with the $\mathrm{KdV}$ equation, the $\mathrm{CH}$ equation has the advantage of admitting peaked 
solitons (peakons) of the form $c e^{-|x-c t|}$, which capture the main feature of the exact traveling wave solutions of greatest height of the governing equations for water waves in irrotational flow [11-13], and modeling wave breaking $[4,6,7]$.

Since the discovery of the $\mathrm{CH}$ equation and its peakon solutions, partial differential equations admitting peakons have attracted much attention in the past two decades. Several equations, such as Degasperi-Procesi (DP) equation [18], V. Novikov equation [24,35], modified CH equation [37] and the Geng-Xue (G-X) equation [19], have similar properties; see [20,38, 40, 42-45] for other multicomponent versions, and $[1,17,21,39]$ for non-integrable peakon equations. These equations are interesting for introducing many challenging problems, including existence, uniqueness, stability and breakdown of solutions, etc. We recall that the $\mathrm{CH}$ (periodic) peakons [8,31] and DP peakons [32] are stable in the sense that their shape is stable under small perturbations, which shows the peakons are detectable. Besides, the peakon interactions were a key ingredient in the development of the theory of continuation after blow-up of global weak solutions of the $\mathrm{CH}$ equation [2,22].

It was shown in $[3,4,9,10]$ that the inverse spectral or scattering approach was a powerful tool to handle Camassa-Holm equation. Particularly, peakon dynamic system of (1.2) was explicitly solved in terms of the classical theory of Stieltjes continued fractions [3] and their interactions were investigated via orthogonal polynomials [4]. Because of the Lax integrability, multipeakons to other peakon equations had also been explicitly formulated via the inverse spectral method (for details, see $[15,16,25,27-29])$. All of these work shows that the mathematics of peakons are closely connected with significant topics and theories in classical analysis, such as sting problem, Stieltjes continued fractions, orthogonal polynomials, Padé approximation, oscillatory kernels, etc.

Back to (1.1), we remark that it is not equivalent to the two-component modified $\mathrm{CH}$ equation [40] for (1.2) can be reduced to (1.1) and the latter cannot. We now review the work on peakons of (1.1) in [45]. The two-component Camassa-Holm system (1.1) admits two-component peakons:

$$
u(x, t)=\sum_{k=1}^{N} m_{k}(t) e^{-\left|x-x_{k}(t)\right|}, \quad v(x, t)=\sum_{k=1}^{N} n_{k}(t) e^{-\left|x-y_{k}(t)\right|},
$$

where $m_{k} n_{k}=0, m_{k}^{2}+n_{k}^{2} \neq 0$. In this case, $m$ and $n$ will be discrete measures with disjoint supports:

$$
m=u-D_{x}^{2} u=2 \sum_{i=1}^{N} m_{i} \delta_{x_{i}}, \quad n=v-D_{x}^{2} v=2 \sum_{j=1}^{N} n_{j} \delta_{y_{j}} .
$$

The peakon ansatz (1.3) satisfies (1.1) if and only if $x_{k}(t), y_{k}(t), m_{k}(t)$ and $n_{k}(t)$ satisfy the following peakon ODEs:

$$
\left\{\begin{array}{l}
\dot{x}_{k}=-\frac{1}{2} \sum_{i, j=1}^{N} m_{i} n_{j}\left(1-\operatorname{sgn}\left(x_{i}-x_{k}\right) \operatorname{sgn}\left(y_{j}-x_{k}\right)\right) e^{-\left|x_{k}-x_{i}\right|-\left|x_{k}-y_{j}\right|} \\
\dot{y}_{k}=-\frac{1}{2} \sum_{i, j=1}^{N} m_{i} n_{j}\left(1-\operatorname{sgn}\left(x_{i}-y_{k}\right) \operatorname{sgn}\left(y_{j}-y_{k}\right) e^{-\left|y_{k}-x_{i}\right|-\left|y_{k}-y_{j}\right|}\right. \\
\dot{m}_{k}=-\frac{1}{2} m_{k} \sum_{i, j=1}^{N} m_{i} n_{j}\left(\operatorname{sgn}\left(y_{j}-x_{k}\right)-\operatorname{sgn}\left(x_{i}-x_{k}\right)\right) e^{-\left|x_{k}-x_{i}\right|-\left|x_{k}-y_{j}\right|} \\
\dot{n}_{k}=\frac{1}{2} n_{k} \sum_{i, j=1}^{N} m_{i} n_{j}\left(\operatorname{sgn}\left(y_{j}-y_{k}\right)-\operatorname{sgn}\left(x_{i}-y_{k}\right)\right) e^{-\left|y_{k}-x_{i}\right|-\left|y_{k}-y_{j}\right|}
\end{array}\right.
$$

for $k=1,2, \ldots, N$. The $1+1$ peakons can be integrated directly, while the peakon ODEs (1.5) with $N>1$ becomes considerably complicated, which makes it difficult to obtain the explicit solution formulas. 
This paper is devoted to deriving the explicit formulas for the solutions to (1.5) using the inverse spectral method. We will only deal with the interlacing case where

$$
u(x, t)=\sum_{k=1}^{K} m_{2 k-1}(t) e^{-\left|x-x_{2 k-1}(t)\right|}, \quad v(x, t)=\sum_{k=1}^{K} n_{2 k}(t) e^{-\left|x-x_{2 k}(t)\right|},
$$

with

$$
\begin{gathered}
m_{2 k-1}, n_{2 k}>0(1 \leq k \leq K), \\
x_{2 k-1}<x_{2 k}(1 \leq k \leq K), \quad x_{2 k}<x_{2 k+1}(1 \leq k \leq K-1) .
\end{gathered}
$$

The work pertinent to this paper is [29,30]. In [29], Lundmark and Szmigielski studied a third order non-selfadjoint boundary value problem coming from the Lax pair(s) of the G-X equation

$$
\begin{aligned}
m_{t}+\left(3 m u_{x}+m_{x} u\right) v & =0, & m & =u-u_{x x}, \\
n_{t}+\left(3 n v_{x}+n_{x} v\right) u & =0, & n & =v-v_{x x},
\end{aligned}
$$

and solved the inverse spectral problem associated with two positive interlacing discrete measures $m$ and $n$ :

$$
m=2 \sum_{k=1}^{K} m_{2 k-1} \delta_{x_{2 k-1}}, \quad n=2 \sum_{k=1}^{K} n_{2 k} \delta_{x_{2 k}} .
$$

The peakons of the form (1.6) were obtained explicitly. The dynamics of interlacing peakons were analyzed in [30].

The rest of our paper is organized as follows. In section 2, we give Lax pairs of (1.1), and set up our approach to deriving explicit formulas for solutions of the peakon ODEs (1.5) in the interlacing case. In Section 3, we study two forward spectral problems associated with positive interlacing discrete measures. In Section 4, we solve the inverse spectral problem via Stieltjes continued fractions, and give two constants of motion. In Section 5, the solutions of the inverse problem construct explicitly the interlacing peakons of (1.1), involving Hankel determinants associated to two different spectral measures.

\section{Preliminaries}

In this section, we recall the Lax integrability of (1.1), state the inverse spectral method applied in our paper.

Equation (1.1) can be derived as the compatibility condition of the linear spectral problem [45]:

$$
\begin{gathered}
\left(\begin{array}{c}
\phi_{1} \\
\phi_{2}
\end{array}\right)_{x}=\frac{1}{2}\left(\begin{array}{cc}
-1 & \lambda m \\
-\lambda n & 1
\end{array}\right)\left(\begin{array}{l}
\phi_{1} \\
\phi_{2}
\end{array}\right), \\
\left(\begin{array}{c}
\phi_{1} \\
\phi_{2}
\end{array}\right)_{t}=-\frac{1}{2}\left(\begin{array}{cc}
2 \lambda^{-2}+\frac{1}{2} Q+\frac{1}{2} P & -\lambda^{-1}\left(u-u_{x}\right)-\frac{1}{2} \lambda m Q \\
\lambda^{-1}\left(v+v_{x}\right)+\frac{1}{2} \lambda n Q & -\frac{1}{2} Q-\frac{1}{2} P
\end{array}\right)\left(\begin{array}{l}
\phi_{1} \\
\phi_{2}
\end{array}\right),
\end{gathered}
$$

where $\lambda$ is a spectral parameter, $m=u-u_{x x}, n=v-v_{x x}, P=u v_{x}-u_{x} v$ and $Q=u v-u_{x} v_{x}$, but also as the compatibility condition of a different linear spectral problem obtained by interlacing $u$ and $v$ : 


$$
\begin{gathered}
\left(\begin{array}{l}
\phi_{1} \\
\phi_{2}
\end{array}\right)_{x}=\frac{1}{2}\left(\begin{array}{cc}
-1 & \lambda n \\
-\lambda m & 1
\end{array}\right)\left(\begin{array}{l}
\phi_{1} \\
\phi_{2}
\end{array}\right), \\
\left(\begin{array}{l}
\phi_{1} \\
\phi_{2}
\end{array}\right)_{t}=-\frac{1}{2}\left(\begin{array}{cc}
2 \lambda^{-2}+\frac{1}{2} Q-\frac{1}{2} P & -\lambda^{-1}\left(v-v_{x}\right)-\frac{1}{2} \lambda n Q \\
\lambda^{-1}\left(u+u_{x}\right)+\frac{1}{2} \lambda m Q & -\frac{1}{2} Q+\frac{1}{2} P
\end{array}\right)\left(\begin{array}{l}
\phi_{1} \\
\phi_{2}
\end{array}\right) .
\end{gathered}
$$

We call (2.2) the twin Lax pair with respect to the Lax pair (2.1) of (1.1).

Under the gauge transformation

$$
\psi_{1}=\phi_{1} e^{\frac{x}{2}}, \quad \psi_{2}=\phi_{2} e^{-\frac{x}{2}},
$$

(2.1a) and (2.2a) become the following simpler counterparts, respectively,

$$
\begin{aligned}
& \left(\begin{array}{l}
\psi_{1} \\
\psi_{2}
\end{array}\right)_{x}=\left(\begin{array}{cc}
0 & \lambda g \\
-\lambda h & 0
\end{array}\right)\left(\begin{array}{l}
\psi_{1} \\
\psi_{2}
\end{array}\right), \quad m=2 g e^{-x}, \quad n=2 h e^{x}, \\
& \left(\begin{array}{l}
\psi_{1} \\
\psi_{2}
\end{array}\right)_{x}=\left(\begin{array}{cc}
0 & \lambda \hat{h} \\
-\lambda \hat{g} & 0
\end{array}\right)\left(\begin{array}{l}
\psi_{1} \\
\psi_{2}
\end{array}\right), \quad m=2 \hat{g} e^{x}, \quad n=2 \hat{h} e^{-x} .
\end{aligned}
$$

We will recover $m$ and $n$ from spectral data obtained by imposing boundary condition

$$
\psi_{1}(-\infty)=\psi_{2}(+\infty)=0
$$

on equations (2.4) and (2.5) when $m$ and $n$ are positive discrete meatures given by (1.9). In this case,

$$
h_{k}=n_{2 k} e^{-x_{2 k}}, \quad g_{k}=m_{2 k-1} e^{x_{2 k-1}}, \quad \hat{h}_{k}=n_{2 k} e^{x_{2 k}}, \quad \hat{g}_{k}=m_{2 k-1} e^{-x_{2 k-1}} .
$$

For later convenience, we make convention that $x_{0}=-\infty, x_{2 K+1}=+\infty$.

To describe our approach specifically, we define the spectral maps as follows:

Definition 2.1 (Forward and inverse spectral maps). Let $\mathrm{P} \subset \mathbb{R}^{4 K}$ be the set of tuples

$$
\mathbf{p}=\left(h_{1}, g_{1}, \hat{h}_{1}, \hat{g}_{1}, \ldots, h_{K}, g_{K}, \hat{h}_{K}, \hat{g}_{K}\right)
$$

satisfying

$$
h_{k}, g_{k}, \hat{h}_{k}, \hat{g}_{k}>0, \quad k=1, \ldots, K .
$$

Let $\mathrm{R} \subset \mathbb{R}^{4 K}$ be the set of tuples

$$
\mathbf{r}=\left(\lambda_{1}^{2}, \cdots, \lambda_{K}^{2} ; \hat{\lambda}_{1}^{2}, \cdots, \hat{\lambda}_{K-1}^{2} ; a_{1}, \ldots, a_{K} ; b_{1}, \ldots, b_{K-1} ; \gamma, \tilde{\gamma}\right)
$$

satisfying

$$
\begin{gathered}
0<\lambda_{1}^{2}<\cdots<\lambda_{K}^{2}, \quad 0<\hat{\lambda}_{1}^{2}<\cdots<\hat{\lambda}_{K-1}^{2}, \quad \gamma, \tilde{\gamma}>0 \\
a_{i}>0, i=1, \ldots, K, \quad b_{j}>0, j=1, \ldots, K-1 .
\end{gathered}
$$

We denote the forward spectral map by

$$
\mathrm{S}: \mathrm{P} \rightarrow \mathrm{R},
$$


which will be given in Section 3, and the the inverse spectral map

$$
\mathrm{T}: \mathrm{R} \rightarrow \mathrm{P}
$$

will be defined in Section 4.

According to the Theorems 3.1, 3.4, 4.4, 4.8 and 4.7, the definition above is suitable. Using the relation (2.7), we have the following relations:

$$
\begin{array}{ll}
x_{2 k}=\frac{1}{2} \ln \left(\frac{\hat{h}_{k}}{h_{k}}\right), & x_{2 k-1}=\frac{1}{2} \ln \left(\frac{g_{k}}{\hat{g}_{k}}\right), \\
n_{2 k}=\left(\hat{h}_{k} h_{k}\right)^{\frac{1}{2}}, & m_{2 k-1}=\left(\hat{g}_{k} g_{k}\right)^{\frac{1}{2}} .
\end{array}
$$

By (2.11a), (2.11b), we can recover the positive interlacing discrete measures (1.9), and give the interlacing peakons (1.6) locally by Theorem 5.1.

Finally, we introduce two types of half-strictly interlacing relation [29], which will be used in Section 3 to describe the solutions of spectral problems.

Definition 2.2. Let $\left(\begin{array}{c}{[1, k]} \\ l\end{array}\right)$ be the set of $l$-element subsets of the integer interval $[1, k]=\{1, \ldots, k\}$, $I, J \in\left(\begin{array}{c}{[1, k]} \\ l\end{array}\right)$ or $I \in\left(\begin{array}{c}{[1, k]} \\ l\end{array}\right), J \in\left(\begin{array}{c}{[1, k]} \\ l-1\end{array}\right)$, then

$$
\begin{aligned}
& I \preccurlyeq J \Leftrightarrow i_{1} \leq j_{1}<i_{2} \leq j_{2}<\cdots<i_{l} \leq j_{l} \quad \text { or } \quad i_{1} \leq j_{1}<i_{2} \leq j_{2}<\cdots<i_{l}, \\
& I \prec J \Leftrightarrow i_{1}<j_{1} \leq i_{2}<j_{2} \leq \cdots \leq i_{l}<j_{l} \quad \text { or } \quad i_{1}<j_{1} \leq i_{2}<j_{2} \leq \cdots \leq i_{l} \text {, }
\end{aligned}
$$

depending on whether $|I|-|J|=0$ or 1 .

\section{Forward Spectral Problem}

In this section, our goal is to define the forward spectral map (2.9). Suppose that $h_{k}, g_{k}, \hat{h}_{k}, \hat{g}_{k}>$ $0(1 \leq k \leq K)$, we obtain the corresponding spectral data, a $4 \mathrm{~K}$-tuple $\mathbf{r} \in \mathrm{R}$. To simplify the structure of the paper, $\tilde{\gamma}$ will be given separately in Section 4 .

\subsection{Spectrum}

Imposing boundary condition (2.6) on (2.4) and (2.5), respectively, we obtain two spectral problems, solving them in the discrete case will give all the $2 K-1$ pairs of eigenvalues.

Definition 3.1 (Eigenvalue). The eigenvalues of the interlacing peakon spectral problem are those values of $\lambda$ for which (2.4) or (2.5), in the case when $m$ and $n$ are positive discrete meatures with interlacing supports, has nontrivial solutions satisfying (2.6). 


\section{Spectral problem I.}

$$
\left(\begin{array}{l}
\psi_{1} \\
\psi_{2}
\end{array}\right)_{x}=\left(\begin{array}{cc}
0 & \lambda g \\
-\lambda h & 0
\end{array}\right)\left(\begin{array}{l}
\psi_{1} \\
\psi_{2}
\end{array}\right), \quad \psi_{1}(-\infty)=\psi_{2}(+\infty)=0
$$

where

$$
g=\sum_{k=1}^{K} g_{k} \delta_{x_{2 k-1}}, \quad h=\sum_{k=1}^{K} h_{k} \delta_{x_{2 k}}
$$

Since $g$ and $h$ are interlacing discrete measures, we have the following facts:

- For any $0 \leq k \leq K, \psi_{1}$ is constant in the interval $x_{2 k-1}<x<x_{2 k+1}$ while $\psi_{2}$ remains constant in $x_{2 k}<x<x_{2 k+2}$.

- For any $1 \leq k \leq K, \psi_{1}$ has a jump $\lambda g_{k} \psi_{2}\left(x_{2 k-1}\right)$ at the point $x_{2 k-1}$ while $\psi_{2}$ has a jump $-\lambda h_{k} \psi_{1}\left(x_{2 k}\right)$ at $x_{2 k}$.

Let $q_{k}=\psi_{1}\left(x_{2 k+1}-\right), p_{k}=\psi_{2}\left(x_{2 k}-\right)$, then the problem (3.1) becomes

$$
\begin{cases}q_{k}-q_{k-1}=\lambda g_{k} p_{k}, & 1 \leq k \leq K, \\ p_{k+1}-p_{k}=-\lambda h_{k} q_{k}, & 1 \leq k \leq K, \\ q_{0}=p_{K+1}=0, & \end{cases}
$$

where $q_{K}=\lim _{x \rightarrow+\infty} \psi_{1}(x), p_{K+1}=\lim _{x \rightarrow+\infty} \psi_{2}(x)$. When $\lim _{x \rightarrow-\infty} \psi_{2}(x)=1$, consider the finite difference problem

$$
\begin{cases}q_{k}-q_{k-1}=\lambda g_{k} p_{k}, & 1 \leq k \leq K, \\ p_{k+1}-p_{k}=-\lambda h_{k} q_{k}, & 1 \leq k \leq K, \\ q_{0}=0, \quad p_{1}=1 . & \end{cases}
$$

Proposition 3.1. The problem (3.3) has a unique solution, which can be given by

$$
\begin{aligned}
& q_{k}=\sum_{l=1}^{k}(-1)^{l+1} \lambda^{2 l-1} \sum_{I \in\left(\begin{array}{c}
{[1, k]} \\
l
\end{array}\right), J \in\left(\begin{array}{c}
{[1, k]} \\
l \preccurlyeq J \\
l-1
\end{array}\right)} g_{I} h_{J}, \\
& p_{k+1}=1+\sum_{l=1}^{k}(-1)^{l} \lambda^{2 l} \sum_{\substack{I, J \in([1, k]) \\
l \\
I \preccurlyeq J}} g_{I} h_{J},
\end{aligned}
$$

where $g_{I}=\prod_{i \in I} g_{i}, h_{J}=\prod_{j \in J} h_{j}$.

Proof. According to (3.3),

$$
\left(\begin{array}{c}
q_{k} \\
p_{k+1}
\end{array}\right)=T_{k}(\lambda) \cdots T_{1}(\lambda)\left(\begin{array}{l}
0 \\
1
\end{array}\right), \quad T_{k}(\lambda)=\left(\begin{array}{cc}
1 & \lambda g_{k} \\
-\lambda h_{k} & 1-\lambda^{2} g_{k} h_{k}
\end{array}\right) .
$$

Using induction, we obtain the conclusion. 


\section{Spectral problem II.}

$$
\left(\begin{array}{l}
\hat{\psi}_{1} \\
\hat{\psi}_{2}
\end{array}\right)_{x}=\left(\begin{array}{cc}
0 & \lambda \hat{h} \\
-\lambda \hat{g} & 0
\end{array}\right)\left(\begin{array}{l}
\hat{\psi}_{1} \\
\hat{\psi}_{2}
\end{array}\right), \quad \hat{\psi}_{1}(-\infty)=\hat{\psi}_{2}(+\infty)=0
$$

where

$$
\hat{h}=\sum_{k=1}^{K} \hat{h}_{k} \delta x_{2 k}, \quad \hat{g}=\sum_{k=1}^{K} \hat{g}_{k} \delta x_{2 k-1}
$$

Let $\hat{q}_{k}=\hat{\psi}_{1}\left(x_{2 k-2}+\right), \hat{p}_{k}=\hat{\psi}_{2}\left(x_{2 k-1}+\right)$, then the problem (3.4) becomes

$$
\begin{cases}\hat{q}_{k+1}-\hat{q}_{k}=\lambda \hat{h}_{k} \hat{p}_{k}, & 1 \leq k \leq K, \\ \hat{p}_{k}-\hat{p}_{k-1}=-\lambda \hat{g}_{k} \hat{q}_{k}, & 1 \leq k \leq K, \\ \hat{q}_{1}=\hat{p}_{K}=0 . & \end{cases}
$$

When $\lim _{x \rightarrow-\infty} \psi_{2}(x)=1$, the finite difference problem

$$
\begin{cases}\hat{q}_{k+1}-\hat{q}_{k}=\lambda \hat{h}_{k} \hat{p}_{k}, & 1 \leq k \leq K, \\ \hat{p}_{k}-\hat{p}_{k-1}=-\lambda \hat{g}_{k} \hat{q}_{k}, & 1 \leq k \leq K, \\ \hat{p}_{0}=1, \quad \hat{q}_{1}=0, & \end{cases}
$$

has a unique solution, which can be written as

$$
\left(\begin{array}{c}
\hat{p}_{K} \\
\hat{q}_{K+1}
\end{array}\right)=\hat{T}_{K}(\lambda) \cdots \hat{T}_{1}(\lambda)\left(\begin{array}{c}
1 \\
0
\end{array}\right), \quad \hat{T}_{k}(\lambda)=\left(\begin{array}{cc}
1 & -\lambda \hat{g}_{k} \\
\lambda \hat{h}_{k} & 1-\lambda^{2} \hat{g}_{k} \hat{h}_{k}
\end{array}\right)
$$

By induction,we have the following proposition as an analogue of Proposition 3.1.

Proposition 3.2. Let $\hat{g}_{I}=\prod_{i \in I} \hat{g}_{i}, \hat{h}_{J}=\prod_{j \in J} \hat{h}_{j}$, then

$$
\begin{gathered}
\hat{p}_{k}=1+\sum_{l=1}^{k-1}(-1)^{l} \lambda^{2 l} \sum_{I \in\left(\begin{array}{c}
{[2, k]} \\
l
\end{array}\right), J \in\left(\begin{array}{c}
{[1, k]} \\
J \gtrless I
\end{array}\right)} \hat{g}_{I} \hat{h}_{J}, \\
\hat{q}_{k+1}=\sum_{l=1}^{k}(-1)^{l+1} \lambda^{2 l-1} \sum_{I \in\left(\begin{array}{c}
{[2, k]} \\
l-1
\end{array}\right), J \in\left(\left[\begin{array}{c}
{[1, k]} \\
J \gtrless I
\end{array}\right)\right.} \hat{g}_{I} \hat{h}_{J} .
\end{gathered}
$$

By Definition 3.1, the spectra of the interlacing peakon spectral problem are zeros of $p_{K+1}$ and $\hat{p}_{K}$. 
Theorem 3.1. If all $g_{k}, h_{k}, \hat{g}_{k}$ and $\hat{h}_{k}$ are positive, then the spectra of (3.1) and (3.4) are non-zero, real, simple and appear in pairs with opposite signs. The eigenvalues can be denoted by

$$
0<\lambda_{1}^{2}<\cdots<\lambda_{K}^{2}, \quad 0<\hat{\lambda}_{1}^{2}<\cdots<\hat{\lambda}_{K-1}^{2} .
$$

The Theorem 3.1 follows from the following algebraic lemma on the Jacobi matrix:

$$
\mathfrak{J}=\left(\begin{array}{cccccc}
0 & d_{1} & 0 & \cdots & 0 & 0 \\
d_{1} & 0 & d_{2} & \cdots & 0 & 0 \\
0 & d_{2} & 0 & \cdots & 0 & 0 \\
\vdots & \vdots & \vdots & \ddots & \vdots & \vdots \\
0 & 0 & 0 & \cdots & 0 & d_{N-1} \\
0 & 0 & 0 & \cdots & d_{N-1} & 0
\end{array}\right), \quad d_{1}, \ldots, d_{N-1}>0 .
$$

Lemma 3.1. Let $\Delta_{k}(\lambda)$ denote the $k$-th order principal minor of $\lambda I-\mathfrak{J}$, with the proviso that $\Delta_{0}=1$, then the roots of $\Delta_{N}(\lambda)=0$ are real and simple.

Proof. It is easy to obtain the recurrence formula

$$
\Delta_{k+1}(\lambda)=\lambda \Delta_{k}(\lambda)-d_{k}^{2} \Delta_{k-1}(\lambda), \quad(k \geq 1),
$$

Therefore, none of adjacent polynomials in the following sequence

$$
\Delta_{N}, \Delta_{N-1}, \ldots, \Delta_{1}, \Delta_{0}
$$

vanishes simultaneously for $\Delta_{0} \equiv 1$. For any eigenvalue $\lambda_{j}, \Delta_{N}\left(\lambda_{j}\right)=0$, then $\Delta_{N-1}\left(\lambda_{j}\right) \neq 0$. Thus, $\operatorname{rank}\left(\lambda_{j} I-\mathfrak{J}\right)=N-1$, so that the geometric multiplicity of $\lambda_{j}$ is 1 . Since $\mathfrak{J}$ is real and symmetric, the multiplicity of $\lambda_{j}$ equals 1 . Therefore, the eigenvalues of $\mathfrak{J}$ are real and simple, which completes the proof.

Remark 3.1. Let $K=\operatorname{diag}(1,-1,1,-1, \ldots)$, then $K^{-1} J K=-J$. Thus

$$
\Delta_{N}(-\lambda)=\operatorname{det}(-\lambda I-\mathfrak{J})=\operatorname{det}\left(K^{-1}(-\lambda I-\mathfrak{J}) K\right)=\operatorname{det}(-\lambda I+\mathfrak{J})=(-1)^{N} \Delta_{N}(\lambda),
$$

taking determinants, we have $\Delta_{N}(\lambda)=(-1)^{N} \Delta_{N}(-\lambda)$. Therefore, when $N$ is even, the roots of $\Delta_{N}(\lambda)=0$ are non-zero, real, simple and appear in pairs with opposite signs.

The proof of Theorem 3.1. According to Lemma 3.1 and its remark, we only need to show that (3.2) and (3.5) are equivalent to some Jacobi matrix eigenvalue problems, respectively.

For the first one, we can rewrite (3.2) as $\bar{J} \bar{\omega}=\lambda \bar{\omega}$, where

$$
\bar{\omega}=\left(-g_{1}^{\frac{1}{2}} p_{1},-h_{1}^{\frac{1}{2}} q_{1}, g_{2}^{\frac{1}{2}} p_{2}, h_{2}^{\frac{1}{2}} q_{2}, \ldots,(-1)^{K} g_{K}^{\frac{1}{2}} p_{K},(-1)^{K} h_{K}^{\frac{1}{2}} q_{K}\right)^{t},
$$

$\bar{J}$ has the form of (3.7) with $N=2 K$ and

$$
\bar{d}_{2 k-1}=\left(g_{k} h_{k}\right)^{-\frac{1}{2}}, \quad \bar{d}_{2 k}=\left(g_{k+1} h_{k}\right)^{-\frac{1}{2}}, \quad k=1,2 \ldots, K .
$$

For the second one, we would like to make some modifications first. Note that (3.5) has nontrivial solutions if and only if the following spectral problem has nontrivial solutions:

$$
\begin{cases}\hat{q}_{k+1}-\hat{q}_{k}=\lambda \hat{h}_{k} \hat{p}_{k}, & 1 \leq k \leq K-1, \\ \hat{p}_{k}-\hat{p}_{k-1}=-\lambda \hat{g}_{k} \hat{q}_{k}, & 2 \leq k \leq K, \\ \hat{q}_{1}=\hat{p}_{K}=0, & \end{cases}
$$


Therefore, (3.5) is equivalent to (3.8), which can be written as

$$
\tilde{J} \tilde{\omega}=\lambda \tilde{\omega},
$$

where

$$
\tilde{\omega}=\left(\hat{h}_{1}^{\frac{1}{2}} \hat{p}_{1}, \hat{g}_{2}^{\frac{1}{2}} \hat{q}_{2},-\hat{h}_{2}^{\frac{1}{2}} \hat{p}_{2},-\hat{g}_{3}^{\frac{1}{2}} \hat{q}_{3}, \ldots,(-1)^{K} \hat{h}_{K-1}^{\frac{1}{2}} \hat{p}_{K-1},(-1)^{K} \hat{g}_{K}^{\frac{1}{2}} \hat{q}_{K}\right)^{t},
$$

$\tilde{J}$ has the form of (3.7) with $N=2 K-2$ and

$$
\tilde{d}_{2 k-1}=\left(\hat{g}_{k+1} \hat{h}_{k}\right)^{-\frac{1}{2}}, \quad \tilde{d}_{2 k}=\left(\hat{g}_{k+1} \hat{h}_{k+1}\right)^{-\frac{1}{2}}, \quad k=1,2 \ldots, K-1 .
$$

This completes the proof of Theorem 3.1.

Remark 3.2. Since $p_{K+1}(0)=\hat{p}_{K}(0)=1$, we have

$$
p_{K+1}=\prod_{j=1}^{K}\left(1-\frac{\lambda^{2}}{\lambda_{j}^{2}}\right), \quad \hat{p}_{K}=\prod_{j=1}^{K-1}\left(1-\frac{\lambda^{2}}{\hat{\lambda}_{j}^{2}}\right) .
$$

\subsection{Weyl functions}

We now define Weyl functions associated to the boundary value problems (3.1) and (3.4) as the following two rational functions:

$$
\begin{gathered}
W(\lambda)=\frac{\psi_{1}(\infty, \lambda)}{\lambda \psi_{2}(\infty, \lambda)}=\frac{q_{K}(\lambda)}{\lambda p_{K+1}(\lambda)}, \\
\hat{W}(\lambda)=\frac{\hat{\psi}_{1}(\infty, \lambda)}{\lambda \hat{\psi}_{2}(\infty, \lambda)}=\frac{\hat{q}_{K+1}(\lambda)}{\lambda \hat{p}_{K}(\lambda)} .
\end{gathered}
$$

By Propositions 3.1, 3.2 and Theorem 3.1, we have the following theorem.

Theorem 3.2. If all $g_{k}, h_{k}, \hat{g}_{k}$ and $\hat{h}_{k}$ are positive, then Weyl functions $W(\lambda), \hat{W}(\lambda)$ have partial fraction decompositions:

$$
\begin{gathered}
W(\lambda)=\sum_{i=1}^{K} \frac{a_{i}}{\lambda_{i}^{2}-\lambda^{2}}, \\
\hat{W}(\lambda)=\gamma+\sum_{j=1}^{K-1} \frac{b_{j}}{\hat{\lambda}_{j}^{2}-\lambda^{2}} .
\end{gathered}
$$

It is elementary to verify that the compatibility conditions of both the Lax pair (2.1) and the twin Lax pair (2.2) with $m$ and $n$ given by (1.4), are equivalent to the non-overlapping peakon ODEs (1.5). Therefore, the interlacing case of (1.5) can induce the evolution of spectral data defined above.

Theorem 3.3 (Evolution). Let $m$ and $n$ be positive measures given by (1.9), $x_{2 k-1}, m_{2 k-1}, x_{2 k}$ and $n_{2 k}$ satisfy (1.5) in the interlacing case, then

$$
\frac{d a_{i}}{d t}=-\frac{a_{i}}{\lambda_{i}^{2}}, \quad \frac{d b_{j}}{d t}=-\frac{b_{j}}{\hat{\lambda}_{j}^{2}}, \quad \frac{d \lambda_{i}}{d t}=0, \quad \frac{d \hat{\lambda}_{j}}{d t}=0, \quad \frac{d \gamma}{d t}=0 .
$$

Hence, $\lambda_{i}, \hat{\lambda}_{j}$ and $\gamma$ are constants of motion. 
Proof. Easy to check that both sides of (2.1b) and (2.2b) vanish when $\psi_{1}(-\infty)=0, \psi_{2}(-\infty)=1$, i.e. the boundary condition imposed when defining the spectral data is consistent with the time evolution.

For $x>x_{2 K}$, substitute $\psi_{1}(x, \lambda)=q_{K}(\lambda), \psi_{2}(x, \lambda)=p_{K+1}$ into $(2.1 \mathrm{~b})$, we obtain

$$
\frac{d q_{K}}{d t}=-\frac{q_{K}}{\lambda^{2}}+\frac{L}{\lambda} p_{K+1}, \quad \frac{d p_{K+1}}{d t}=0, \quad L=\sum_{k=1}^{K} g_{k}
$$

Similarly, substituting $\hat{\psi}_{1}(x, \lambda)=\hat{q}_{K+1}, \hat{\psi}_{2}(x, \lambda)=\hat{p}_{K}$ into (2.2b) leads to

$$
\frac{d \hat{q}_{K+1}}{d t}=-\frac{\hat{q}_{K+1}}{\lambda^{2}}+\frac{\hat{L}}{\lambda} \hat{p}_{K}, \quad \frac{d \hat{p}_{K}}{d t}=0, \quad \hat{L}=\sum_{k=1}^{K} \hat{h}_{k}
$$

Thus, the eigenvalues $\lambda_{i}, \hat{\lambda}_{j}$ are time invariant, and

$$
\begin{aligned}
& \frac{d W}{d t}=-\frac{W}{\lambda^{2}}+\frac{L}{\lambda^{2}}, \\
& \frac{d \hat{W}}{d t}=-\frac{\hat{W}}{\lambda^{2}}+\frac{\hat{L}}{\lambda^{2}} .
\end{aligned}
$$

Taking residues of both sides of (3.16) and (3.17) at $\lambda_{i}, \hat{\lambda}_{j}$, respectively, we obtain the evolution of $a_{i}, b_{j}$. Computing $\lim _{\lambda \rightarrow \infty} \frac{d \hat{W}}{d t}$ leads to $\frac{d \gamma}{d t}=0$, i.e. $\gamma$ is a constant of motion.

Corollary 3.1. The quantities

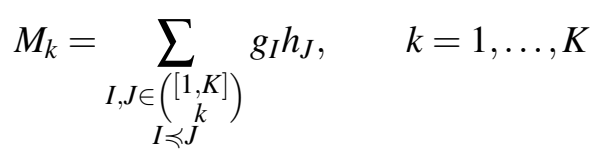

and

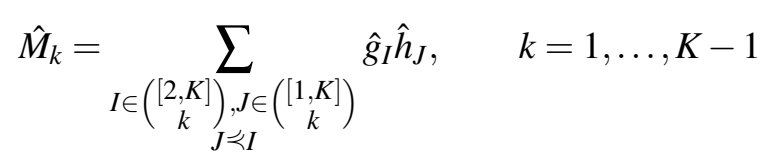

form $2 K-1$ constants of motion for the peakon ODEs (1.5) in the interlacing case.

Proof. From (3.14) and (3.15), the coefficients of $p_{K+1}$ and $\hat{p}_{K}$ are time invariant, therefore, the conclusion follows Propositions 3.1 and 3.2.

Theorem 3.4. If all $g_{k}, h_{k}, \hat{g}_{k}$ and $\hat{h}_{k}$ are positive, then $a_{i}, b_{j}$ in Theorem 3.2 are positive.

The proof of Theorem 3.4 is closely related to the following results of J. Moser on Jacobi spectral problem $[33,34]$. 
Lemma 3.2 (J. Moser). Let $f(\lambda)$ be the $(N, N)$ entry of $(\lambda I-\mathfrak{J})^{-1}$, i.e.

$$
f(\lambda)=\Delta_{N-1}(\lambda) / \Delta_{N}(\lambda)
$$

then $f(\lambda)$ has partial fraction decomposition

$$
f(\lambda)=\sum_{j=1}^{l} \frac{2 \lambda r_{j}}{\lambda^{2}-\lambda_{j}^{2}}+\kappa_{N} \frac{r_{l+1}}{\lambda}, \quad r_{j}>0,
$$

where $l=[N / 2], \kappa_{N}=1$ when $N$ is odd and $\kappa_{N}=0$ when $N$ is even.

The proof of Theorem 3.4. Due to the equivalence established in the proof of Theorem 3.1, we can obtain the relations between Weyl functions of interlacing peakon problems (3.1) and (3.4) and some rational functions admitting the properties above.

There is a bit of difficulty in proving that $b_{j}>0$, we deal with it first. Let $\tilde{e}=(0, \ldots, 0,1)$ be a row vector of dimension $2 K-2$, then (3.8) without $\hat{p}_{K}=0$ can be written as

$$
\tilde{e}^{t}(-1)^{K+1} \hat{p}_{K} / \hat{g}_{K}^{\frac{1}{2}}=(\lambda I-\tilde{J}) \tilde{\omega},
$$

Denote the $(2 K-2,2 K-2)$ entry of $(\lambda I-\widetilde{J})^{-1}$ by $\tilde{W}(\lambda)$, then $-\hat{g}_{K} \hat{q}_{K} / \hat{p}_{K}=\tilde{W}(\lambda)$. Hence,

$$
\tilde{W}(\lambda)=-\lambda \hat{g}_{K} \hat{W}(\lambda)+\lambda \hat{h}_{K} \hat{g}_{K}
$$

By Lemma 3.2, $\tilde{W}(\lambda)=\sum_{j=1}^{K-1} \frac{2 \lambda \tilde{r}_{j}}{\lambda^{2}-\hat{\lambda}_{j}^{2}}\left(\tilde{r}_{j}>0\right)$, taking residue of (3.19) at $\hat{\lambda}_{j}$ leads to $2 \tilde{r}_{j}=\hat{g}_{K} b_{j}$, therefore, $b_{j}>0$.

The proof of $a_{i}>0$ is analogous. Denote the $(2 K, 2 K)$ entry of $(\lambda I-\bar{J})^{-1}$ by $\bar{W}(\lambda)$, then $\bar{W}(\lambda)=-\lambda h_{K} W(\lambda)$, which leads to $a_{i}>0$.

Remark 3.3. Theorems 3.1 and 3.4 can also be obtained in the theory of orthogonal polynomials.

Remark 3.4. $\tilde{\gamma}$ will be given as certain duality of $\gamma$ and shown to be time invariant at the end of Section 4, and $\gamma, \tilde{\gamma}>0$ will follow from Theorem 4.3.

We have now completed the spectral characterization of the boundary value problems (3.1) and (3.4), and the construction of the forward spectral map (2.9).

\section{Inverse Spectral Problem}

In this section, with the help of Stieltjes continued fractions, we will solve the inverse spectral problem of recovering two pairs of discrete interlacing measures $g, h$ and $\hat{g}, \hat{h}$ from the spectral data $\left\{\lambda_{i}^{2}, \hat{\lambda}_{j}^{2}, a_{i}, b_{j}, \gamma, \tilde{\gamma}\right\}$ that they give rise to. $\hat{W}(\lambda)$.

We first introduce two theorems to characterize the properties of Weyl functions $W(\lambda)$ and 
Theorem 4.1. Let $z=-\lambda^{2}, \mu_{i}=\lambda_{i}^{2}, v_{j}=\hat{\lambda}_{j}^{2}$, define two spectral measures as follows:

$$
d \alpha(\mu)=\sum_{i=1}^{K} a_{i} \delta_{\mu_{i}}, \quad d \beta(v)=\sum_{j=1}^{K-1} b_{j} \delta_{v_{j}},
$$

then $W(\lambda)$ and $\hat{W}(\lambda)$ admit the following integral representations

$$
W(\lambda)=\int \frac{d \alpha(\mu)}{z+\mu}, \quad \hat{W}(\lambda)=\gamma+\int \frac{d \beta(v)}{z+v} .
$$

Theorem 4.2. Weyl functions $W(\lambda)$ and $\hat{W}(\lambda)$ have continued fraction expansions:

$$
W(\lambda)=\frac{1}{-\lambda^{2} h_{K}+\frac{1}{g_{K}+\cdots+\frac{1}{-\lambda^{2} h_{1}+\frac{1}{g_{1}}}}},
$$

Proof. Using the recursive relations (3.6), we can obtain (4.2) by induction. In fact,

$$
\frac{\hat{q}_{k+1}}{\lambda \hat{p}_{k}}=\frac{\hat{q}_{k}+\lambda \hat{h}_{k} \hat{p}_{k}}{\lambda \hat{p}_{k}}=\hat{h}_{k}+\frac{1}{\frac{\lambda\left(\hat{p}_{k-1}-\lambda \hat{g}_{k} \hat{q}_{k}\right)}{\hat{q}_{k}}}=\hat{h}_{k}+\frac{1}{-\lambda^{2} \hat{g}_{k}+\frac{1}{\frac{\hat{q}_{k}}{\lambda \hat{p}_{k-1}}}} .
$$

Taking account of $\hat{q}_{1}=0$, we have the continued fraction expansion (4.2). The proof of (4.1) is analogues.

The following results of T. Stieltjes [41] will recover $h_{k}, g_{k}(k=1, \ldots, K), \hat{h}_{1}, \hat{h}_{k}, \hat{g}_{k}(k=$ $2, \ldots, K-1), \hat{g}_{K}$ by elementary functions of spectral data, and verify that $\hat{h}_{K}$ and $\hat{g}_{1}$ are constant.

Theorem 4.3 (Stieltjes). Suppose $F(z)$ is a rational function admitting the following integral representation:

$$
F(z)=c+\int \frac{d \mu(x)}{x+z}
$$

here, $d \mu(x)$ is the Stieltjes measure corresponding to the increasing piecewise constant function $\mu(x)$ with finite jumps on $\mathbb{R}$, then $F(z)$ can be developed in a finite Stieltjes continued fraction:

$$
F(z)=c+\frac{1}{z a_{1}+\frac{1}{a_{2}+\frac{1}{z a_{3}+\cdots}}}, \quad a_{i}>0
$$


conversely, any rational function with this type of continued fraction admits integral representation of the form (4.3). Particularly, when $d \mu=\sum_{j=1}^{N} b_{j} \delta_{\lambda_{j}}$, set

$$
H_{k}^{l}=\operatorname{det}\left(A_{i+j+l}\right)_{i, j=0}^{k-1}(l \geq 0), \quad A_{k}=\int_{-\infty}^{+\infty} x^{k} d \mu(x)
$$

with the proviso that $H_{0}^{l}=H_{0}^{0}=1$, if $H_{i}^{0}, H_{j}^{1}>0(i, j \leq N)$, then

$$
a_{2 k}=\frac{\left(H_{k}^{0}\right)^{2}}{H_{k}^{1} H_{k-1}^{1}}, \quad a_{2 k+1}=\frac{\left(H_{k}^{1}\right)^{2}}{H_{k}^{0} H_{k+1}^{0}} .
$$

By Theorem 4.3, $\gamma=\hat{h}_{K}$. Taking account of Theorem 3.3 and the relation (2.7), we have the following theorem.

Theorem 4.4. $\gamma>0, \hat{h}_{K}$ is a constant of motion, and

$$
\hat{h}_{K}=n_{2 K}(0) \exp \left(x_{2 K}(0)\right)>0 .
$$

To recover $h_{k}, g_{k}(k=1, \ldots, K)$ and $\hat{h}_{1}, \hat{g}_{2}, \ldots, \hat{h}_{K-1}, \hat{g}_{K}$, we need to ensure the positivity of some Hankel determinants. The following lemma follows from Heine's formula on $k \times k$ Hankel determinant of moments.

\section{Lemma 4.1.}

$$
H_{k}^{l}=\sum_{J \in\left(\begin{array}{c}
{[1, N]} \\
k
\end{array}\right)} b_{J} \lambda_{J}^{l} \Delta_{J}^{2}, \quad 0 \leq k \leq N
$$

where

$$
b_{J}=\prod_{j \in J} b_{j}, \quad \lambda_{J}^{l}=\prod_{j \in J} \lambda_{j}^{l}, \quad \Delta_{J}^{2}=\prod_{i, j \in J, i<j}\left(\lambda_{j}-\lambda_{i}\right)^{2} .
$$

Remark 4.1. Lemma 4.1 together with the properties of spectral data given by Theorems 3.1 and 3.4 shows that $H_{i}^{l}>0(0 \leq i \leq K), \quad \hat{H}_{j}^{l}>0(0 \leq j \leq K-1)$.

Applying Theorem 4.3 to $W(\lambda)$ recovers $h_{k}, g_{k}(k=1, \ldots, K)$.

\section{Theorem 4.5.}

$$
g_{K+1-k}=\frac{\left(H_{k}^{0}\right)^{2}}{H_{k}^{1} H_{k-1}^{1}}, \quad h_{K+1-k}=\frac{\left(H_{k-1}^{1}\right)^{2}}{H_{k}^{0} H_{k-1}^{0}}, \quad k=1, \ldots, K,
$$

where $H_{k}^{l}=\operatorname{det}\left(A_{i+j+l}\right)_{i, j=0}^{k-1}(l \geq 0), A_{k}=\int \mu^{k} d \alpha(\mu)=\sum_{j=1}^{K} \lambda_{j}^{2 k} a_{j}$.

Apply Theorem 4.3 to $\hat{W}(\lambda)-\gamma\left(\right.$ or $\hat{W}(\lambda)$ ), we have $\hat{h}_{1}, \hat{g}_{2}, \ldots, \hat{h}_{K-1}$ and $\hat{g}_{K}$.

\section{Theorem 4.6.}

$$
\hat{h}_{K-k}=\frac{\left(\hat{H}_{k}^{0}\right)^{2}}{\hat{H}_{k}^{1} \hat{H}_{k-1}^{1}}, \quad \hat{g}_{K+1-k}=\frac{\left(\hat{H}_{k-1}^{1}\right)^{2}}{\hat{H}_{k}^{0} \hat{H}_{k-1}^{0}}, \quad k=1, \ldots, K-1,
$$

where $\hat{H}_{k}^{l}=\operatorname{det}\left(\hat{A}_{i+j+l}\right)_{i, j=0}^{k-1}(l \geq 0), \quad \hat{A}_{k}=\int v^{k} d \beta(v)=\sum_{j=1}^{K-1} \hat{\lambda}_{j}^{2 k} b_{j}$.

The following theorem follows from Remark 4.1 and the two theorems above. 
Theorem 4.7. $g_{1}, h_{1}, \ldots, g_{K}, h_{K}, \hat{h}_{1}, \hat{g}_{2}, \ldots, \hat{h}_{K-1}, \hat{g}_{K}$ given by (4.7) and (4.8) are always positive.

We will finish this section by defining $\tilde{\gamma}>0$ and recovering $\hat{g}_{1}$.

Let us consider the spectral problem (3.1) again, and define another Weyl function $\hat{W}^{*}(\lambda)$. To avoid confusion, we rewrite (3.1) as follows:

$$
\left(\begin{array}{c}
\tilde{\psi}_{1} \\
\tilde{\psi}_{2}
\end{array}\right)_{x}=\left(\begin{array}{cc}
0 & \lambda \hat{h} \\
-\lambda \hat{g} & 0
\end{array}\right)\left(\begin{array}{l}
\tilde{\psi}_{1} \\
\tilde{\psi}_{2}
\end{array}\right), \quad \tilde{\psi}_{1}(-\infty)=\tilde{\psi}_{2}(+\infty)=0
$$

Let $\tilde{q}_{k}=\tilde{\psi}_{1}\left(x_{2 k}-\right), \tilde{p}_{k}=\tilde{\psi}_{2}\left(x_{2 k+1}-\right)$ and $\tilde{\psi}_{1}(+\infty)=1$, consider the finite difference equation

$$
\begin{cases}\tilde{q}_{k+1}-\tilde{q}_{k}=\lambda \hat{h}_{k} \tilde{p}_{k}, & 1 \leq k \leq K, \\ \tilde{p}_{k}-\tilde{p}_{k-1}=-\lambda \hat{g}_{k} \tilde{q}_{k}, & 1 \leq k \leq K, \\ \tilde{p}_{K}=0, \quad \tilde{q}_{K+1}=1 . & \end{cases}
$$

Using (4.10), we have

$$
\left(\begin{array}{c}
\tilde{p}_{0} \\
\tilde{q}_{1}
\end{array}\right)=\hat{S}(\lambda)^{-1}\left(\begin{array}{l}
0 \\
1
\end{array}\right), \quad \hat{S}(\lambda):=\hat{T}_{K}(\lambda) \cdots \hat{T}_{1}(\lambda)
$$

Note that $\hat{S}(\lambda)^{-1}=\left(\begin{array}{cc}\hat{S}_{22} & -\hat{S}_{12} \\ -\hat{S}_{21} & \hat{S}_{11}\end{array}\right)$, thus, $\tilde{q}_{1}=\lim _{x \rightarrow-\infty} \tilde{\psi}_{1}(x)=0$ if and only if $\hat{S}_{11}=0$. Hence, (4.9) really has the same spectra as (3.4).

Define Weyl function of spectral problem (4.9) by the second column of $\hat{S}(\lambda)^{-1}$,

$$
\hat{W}^{*}(\lambda)=-\frac{\hat{S}_{12}(\lambda)}{\lambda \hat{S}_{11}(\lambda)}
$$

Recall that

$$
\hat{W}^{(\lambda)}=\frac{\hat{q}_{K+1}(\lambda)}{\lambda \hat{p}_{K}(\lambda)}=\frac{\hat{S}_{21}(\lambda)}{\lambda \hat{S}_{11}(\lambda)}
$$

and

$$
\operatorname{deg} \hat{S}(\lambda)=\left(\begin{array}{cc}
2 K-2 & 2 K-1 \\
2 K-1 & 2 K
\end{array}\right)
$$

Therefore, the partial fraction decompositions of Weyl functions $\hat{W}(\lambda)$ and $\hat{W}^{*}(\lambda)$ have the same form, and we can set

$$
\hat{W}^{*}(\lambda)=\tilde{\gamma}+\sum_{j=1}^{K-1} \frac{b_{j}^{*}}{\hat{\lambda}_{j}^{2}-\lambda^{2}}
$$

The proof of $b_{j}^{*}>0$. We show $b_{j}^{*}>0$ by using the positivity of $b_{j}$. 
Obviously, $\operatorname{det} \hat{S}=\operatorname{det} \hat{T}_{K} \cdots \operatorname{det} \hat{T}_{1}=1$, since $\pm \hat{\lambda}_{j}$ are zeros of $\hat{S}_{11}$, we obtain

$$
\hat{S}_{12}\left(\hat{\lambda}_{j}\right) \hat{S}_{21}\left(\hat{\lambda}_{j}\right)=\hat{S}_{12}\left(-\hat{\lambda}_{j}\right) \hat{S}_{21}\left(-\hat{\lambda}_{j}\right)=-1 \text {. }
$$

Easy to know that

$$
b_{j}=-2 \hat{S}_{21}\left( \pm \hat{\lambda}_{j}\right) / \hat{S}_{11}^{\prime}\left( \pm \hat{\lambda}_{j}\right), \quad b_{j}^{*}=2 \hat{S}_{12}\left( \pm \hat{\lambda}_{j}\right) / \hat{S}_{11}^{\prime}\left( \pm \hat{\lambda}_{j}\right),
$$

therefore,

$$
b_{j} b_{j}^{*}=\left(\frac{2}{\hat{S}_{11}^{\prime}\left( \pm \hat{\lambda}_{j}\right)}\right)^{2} .
$$

By Theorem 3.1, $\hat{S}_{11}^{\prime}\left( \pm \hat{\lambda}_{j}\right) \neq 0$, which leads to $b_{j}^{*}>0$.

Using the positivity of $b_{j}^{*}$, we can write $\hat{W}^{*}$ as the Stieltjes transformation of a positive discrete measure

$$
\hat{W}^{*}(\lambda)=\tilde{\gamma}+\int \frac{d \beta^{*}(v)}{z+v}, \quad \beta^{*}(v)=\sum_{j=1}^{K-1} b_{j}^{*} \delta_{v_{j}} .
$$

Due to the fact that (1.1) can also be derived as the compatibility condition of the following linear spectral problem:

$$
\begin{gathered}
\left(\begin{array}{c}
\phi_{1} \\
\phi_{2}
\end{array}\right)_{x}=\frac{1}{2}\left(\begin{array}{cc}
-1 & \lambda n \\
-\lambda m & 1
\end{array}\right)\left(\begin{array}{l}
\phi_{1} \\
\phi_{2}
\end{array}\right), \\
\left(\begin{array}{c}
\phi_{1} \\
\phi_{2}
\end{array}\right)_{t}=-\frac{1}{2}\left(\begin{array}{cc}
\frac{1}{2} Q-\frac{1}{2} P & -\lambda^{-1}\left(v-v_{x}\right)-\frac{1}{2} \lambda n Q \\
\lambda^{-1}\left(u+u_{x}\right)+\frac{1}{2} \lambda m Q & -2 \lambda^{-2}-\frac{1}{2} Q+\frac{1}{2} P
\end{array}\right)\left(\begin{array}{l}
\phi_{1} \\
\phi_{2}
\end{array}\right),
\end{gathered}
$$

we have the following theorem.

Theorem 4.8. $\tilde{\gamma}>0, \hat{g}_{1}$ is a constant of motion and

$$
\hat{g}_{1}=m_{1}(0) \exp \left(-x_{1}(0)\right) .
$$

Proof. Using (4.10), $\hat{W}^{*}(\lambda)$ can be written as the following continued fraction

$$
\hat{W}^{*}(\lambda)=\hat{g}_{1}+\frac{1}{-\lambda^{2} \hat{h}_{1}+\frac{1}{\hat{g}_{2}+\cdots+\frac{1}{-\lambda^{2} \hat{h}_{K-1}+\frac{1}{\hat{g}_{K}}}} .}
$$

Thus, by Theorem 4.3, $\tilde{\gamma}=\hat{g}_{1}>0$.

Easy to check that (1.5) is equivalent to the compatibility condition of (4.12) with $m, n$ given by (1.4), and both sides of (4.12b) vanish when $\psi_{1}(+\infty)=1, \psi_{2}(+\infty)=0$. Hence, for $x<x_{1}$, substituting $\tilde{\psi}_{1}(x, \lambda)=\tilde{q}_{1}, \tilde{\psi}_{2}(x, \lambda)=\tilde{p}_{0}$ into $(4.12 \mathrm{~b})$, we obtain

$$
\frac{d \tilde{p}_{0}}{d t}=\frac{\tilde{p}_{0}}{\lambda^{2}}-\frac{\tilde{L}}{\lambda} \tilde{q}_{1}, \quad \frac{d \tilde{q}_{1}}{d t}=0, \quad \tilde{L}=\sum_{k=1}^{K} \hat{g}_{k} .
$$

Therefore, $\tilde{\gamma}$ in (4.11) is a constant of motion, which completes the proof. 
F.F. Dong and L.J. Zhou / Peakon Problem of an Integrable Two-component Camassa-Holm System

Remark 4.2. In the proof above, we obtain the evolution of $b_{j}^{*}$ :

$$
\frac{d b_{j}^{*}}{d t}=\frac{b_{j}^{*}}{\hat{\lambda}_{j}^{2}}
$$

Apply Theorem 4.3 to $\hat{W}^{*}(\lambda)$, we can obtain the following explicit formulas for $\hat{h}_{1}, \hat{h}_{k}, \hat{g}_{k}(k=$ $2, \ldots, K-1)$ and $\hat{g}_{K}$ :

$$
\hat{h}_{k}=\frac{\left(\tilde{H}_{k-1}^{1}\right)^{2}}{\tilde{H}_{k-1}^{0} \tilde{H}_{k}^{0}}, \quad \hat{g}_{k+1}=\frac{\left(\tilde{H}_{k}^{0}\right)^{2}}{\tilde{H}_{k}^{1} \tilde{H}_{k-1}^{1}}, \quad k=1, \ldots, K-1,
$$

where $\tilde{H}_{k}^{l}=\operatorname{det}\left(\tilde{A}_{i+j+l}\right)_{i, j=0}^{k-1}(l \geq 0), \quad \tilde{A}_{k}=\int v^{k} d \beta^{*}(v)=\sum_{j=1}^{K-1} \hat{\lambda}_{j}^{2 k} b_{j}^{*}$.

\section{Interlacing Peakons}

By Remark 4.1, together with the relations (2.11a) and (2.11b), we have the explicit formulas for positive interlacing peakons.

Theorem 5.1. The new two-component Camassa-Holm system (1.1) admits positive interlacing peakons:

$$
u(x, t)=\sum_{k=1}^{K} m_{2 k-1}(t) e^{-\left|x-x_{2 k-1}(t)\right|}, \quad v(x, t)=\sum_{k=1}^{K} n_{2 k}(t) e^{-\left|x-x_{2 k}(t)\right|},
$$

where

$$
\begin{aligned}
& x_{1}=\frac{1}{2} \ln \left(\frac{e^{x_{1}(0)}}{m_{1}(0)} \frac{\left(H_{K}^{0}\right)^{2}}{H_{K}^{1} H_{K-1}^{1}}\right), \quad m_{1}=\left(m_{1}(0) e^{-x_{1}(0)} \frac{\left(H_{K}^{0}\right)^{2}}{H_{K}^{1} H_{K-1}^{1}}\right)^{\frac{1}{2}}, \\
& x_{2 K}=\frac{1}{2} \ln \left(n_{2 K}(0) e^{x_{2 K}(0)} A_{0}\right), \quad n_{2 K}=\left(n_{2 K}(0) e^{x_{2 K}(0)} / A_{0}\right)^{\frac{1}{2}}, \\
& x_{2 K-2 k+1}=\frac{1}{2} \ln \left(\frac{\left(H_{k}^{0}\right)^{2}}{H_{k}^{1} H_{k-1}^{1}} \frac{\hat{H}_{k}^{0} \hat{H}_{k-1}^{0}}{\left(\hat{H}_{k-1}^{1}\right)^{2}}\right), \\
& m_{2 K-2 k+1}=H_{k}^{0} \hat{H}_{k-1}^{1}\left(H_{k}^{1} H_{k-1}^{1} \hat{H}_{k-1}^{0} \hat{H}_{k}^{0}\right)^{-\frac{1}{2}}, \quad 1 \leq k \leq K-1 \\
& x_{2 K-2 k+2}=\frac{1}{2} \ln \left(\frac{\left(\hat{H}_{k-1}^{0}\right)^{2}}{\hat{H}_{k-1}^{1} \hat{H}_{k-2}^{1}} \frac{H_{k}^{0} H_{k-1}^{0}}{\left(H_{k-1}^{1}\right)^{2}}\right), \\
& n_{2 K-2 k+2}=H_{k-1}^{1} \hat{H}_{k-1}^{0}\left(\hat{H}_{k-1}^{1} \hat{H}_{k-2}^{1} H_{k}^{0} H_{k-1}^{0}\right)^{-\frac{1}{2}}, \quad 2 \leq k \leq K
\end{aligned}
$$

with

$$
\begin{aligned}
& H_{k}^{l}=\operatorname{det}\left(A_{i+j+l}\right)_{i, j=0}^{k-1}(l \geq 0), \quad A_{k}=\int \mu^{k} d \alpha(\mu)=\sum_{i=1}^{K} \lambda_{i}^{2 k} a_{i}, \\
& \hat{H}_{k}^{l}=\operatorname{det}\left(\hat{A}_{i+j+l}\right)_{i, j=0}^{k-1}(l \geq 0), \quad \hat{A}_{k}=\int v^{k} d \beta(v)=\sum_{j=1}^{K-1} \hat{\lambda}_{j}^{2 k} b_{j},
\end{aligned}
$$


and

$$
a_{i}=a_{i}(0) \exp \left(-\frac{t}{\lambda_{i}^{2}}\right), \quad b_{j}=b_{j}(0) \exp \left(-\frac{t}{\hat{\lambda}_{j}^{2}}\right), \quad a_{i}(0), b_{j}(0)>0 .
$$

Remark 5.1. The formulas in Theorem 5.1 give positive interlacing peakons globally if and only if

$$
x_{2 k-1}<x_{2 k}(1 \leq k \leq K), \quad x_{2 k}<x_{2 k+1}(1 \leq k \leq K-1) .
$$

Due to the relations (2.11a) and (2.11b), (5.1) is equivalent to

$$
g_{k} h_{k}<\hat{g}_{k} \hat{h}_{k}(1 \leq k \leq K), \quad \hat{g}_{k+1} \hat{h}_{k}<g_{k+1} h_{k}(1 \leq k \leq K-1) .
$$

For $K=1$, since

$$
m_{1}(0) n_{2}(0) e^{x_{2}(0)}>e^{x_{1}(0)} / \lambda_{1}^{2}, \quad \lambda_{1}^{2}=\left(g_{1}(0) h_{1}(0)\right)^{-1},
$$

(5.1) holds for all $t$ if $x_{1}(0)<x_{2}(0)$. When $K>1$, (5.1) holds only for the spectral data from certain subset of $\mathrm{R}$, which will be studied in the future.

\section{Conclusion and Discussions}

In this paper, we have formulated and solved the peakon problem arising from the explicit construction of interlacing peakons for the new two-component Camassa-Holm system derived by Xia and Qiao, and obtained the explicit formulas for positive interlacing peakons locally. As a generalization of the $\mathrm{CH}$ equation and modified $\mathrm{CH}$ equation (a special case of (1.1) when $v=-2 u$ ), there are some new features and complexity of the solutions. Further studies on the global existence and other properties of positive interlacing peakons to (1.1) will be developed.

In our paper, the classical result of T. Stieltjes on continued fractions allow us to solve the inverse problem associated to the positive interlacing peakons in the case $N=2 K$, how about $N=2 K+1$, where the number of peakons in $u$ and $v$ is not equal, for example, the measure $m$ is supported on the sites $x_{1}, x_{3}, \ldots, x_{2 K+1}$ ? In [29,30], Lundmark and Szmigielski use the inverse spectral method to derive explicit formulas for the interlacing $K+K$ peakons, and explore their dynamical properties. In their work, a sort of symmetry in the case $N=2 K$ is key to the inverse problem, for the interlacing case $N=2 K+1$ or more general one, certain limiting procedures is needed. However, in our case, the interlacing peakons for $N=2 K+1$ can also be explicitly constructed, the operation is similar to the case $N=2 K$, and somewhat easier.

\section{Acknowledgments}

This work was supported by the National Natural Science Foundation of China [NSFC11301398] and the Fundamental Research Funds for the Central Universities.

\section{References}

[1] S.C. Anco, P.L.D. Silva and I.L. Freire, A family of wave-breaking equations generalizing the CamassaHolm and Novikov equations, J. Math. Phys. 56 (2015) 091506.

[2] A. Bressan and A. Constantin, Global conservative solutions of the Camassa-Holm equation, Arch. Ration. Mech. Anal. 183 (2007), 215-239.

[3] R. Beals, D.H. Sattinger and J. Szmigielski, Multipeakons and a theorem of Stieltjes, Inverse Problems 15 (1) (1999) $1-4$. 
[4] R. Beals, D.H. Sattinger and J. Szmigielski, Multipeakons and the classical moment problem, $A d v$. Math. 154 (2000) 229-257.

[5] R. Camassa and D.D. Holm, An integrable shallow water equation with peaked solitons, Phys. Rev. Lett. 71 (1993) 1661-1664.

[6] R. Camassa, D.D. Holm and A.N.W. Hone, A new integrable shallow water equation, Adv. Appl. Mech. 31 (1994) 1-33.

[7] A. Constantin and J. Escher, Wave breaking for nonlinear nonlocal shallow water equations, Acta Math. 181 (2) (1998) 229-243.

[8] A. Constantin and W. Strauss, Stability of peakons, Comm. Pure Appl. Math. 53 (2000), 603-610.

[9] A. Constantin, On the Scattering Problem for the Camassa-Holm Equation, Proc. R. Soc. Lond. A 457 (2001) 953-970.

[10] A. Constantin, On the inverse spectral problem for the Camassa-Holm equation, J. Funct. Anal. 155 (1998) 352-363.

[11] A. Constantin, The trajectories of particles in Stokes waves, Invent. Math. 166 (2006), 523-535.

[12] A. Constantin and J. Escher, Particle trajectories in solitary water waves, Bull. Amer. Math. Soc. 44 (2007), 423-431.

[13] A. Constantin, Particle trajectories in extreme Stokes waves. IMA J. Appl. Math. 77 (2012), 293-307.

[14] A. Constantin and D. Lannes, The hydrodynamical relevance of the Camassa-Holm and DegasperisProcesi equations, Arch. Ration. Mech. Anal. 192 (2009) 165-186.

[15] X.K. Chang, X.B. Hu and J. Szmigielski, Multipeakons of a two-component modified Camassa-Holm equation and the relation with the finite Kac-van Moerbeke lattice, Adv. Math. 299 (2016) 1-35.

[16] X. Chang and J. Szmigielski, Lax integrability and the peakon problem for the modified Camassa-Holm equation, Commun. Math. Phys. (2018) https://doi.org/10.1007/s00220-017-3076-6

[17] A. Degasperis, D.D. Holm and A.N.W. Hone, Integrable and non-integrable equationswith peakons, in Nonlinear Physics: Theory and Experiment, eds. M. J. Ablowitz, M. Boiti, F. Pempinelli, and B. Prinari, Vol. II (Gallipoli, 2002), World Scientific Publishing, New Jersey, 2003, pp. 37-43.

[18] A. Degasperis, D.D. Holm and A.N.W. Hone, A new integral equation with peakon solutions, Theor. Math. Phys. 133 (2002) 1463-1474.

[19] X. Geng and B. Xue, An extension of integrable peakon equations with cubic nonlinearity, Nonlinearity. 22 (8) (2009) 1847-1856.

[20] X. Geng and B. Xue, A three-component generalization of Camassa-Holm equation with N-peakon solutions, Adv. Math. 226 (1) (2011) 827-839.

[21] A. Himonas and D. Mantzavinos, An ab-family of equations with peakon traveling waves, Proc. Amer. Math. Soc. 144 (9) (2016) 3797-3811.

[22] H. Holden and X. Raynaud, Global conservative solutions of the Camassa-Holm equation-a Lagrangian point of view, Comm. Partial Differential Equations 32 (2007), 1511-1549.

[23] Q. Hu and Z. Qiao, Persistence Properties and Unique Continuation for a Dispersionless TwoComponent Camassa-Holm System with Peakon and Weak Kink Solutions, Discrete Contin. Dyn. Syst. A 36 (5) (2015) 2613-2625.

[24] A.N.W. Hone and J.P. Wang, Integrable peakon equations with cubic nonlinearity, J. Phys. A 41 (2008) 372002.

[25] A.N.W. Hone, H. Lundmark and J. Szmigielski, Explicit multipeakon solutions of an Novikov's cubically nonlinear integrable Camassa-Holm type equation, Dyn. Partial Differ. Equ. 6 (3) (2009) 253-289.

[26] R.S. Johnson, Camassa-Holm, Korteweg-de Vries and related models for water waves, J. Fluid Mech. 457 (2002) 63-82.

[27] H. Lundmark and J. Szmigielski, Multi-peakon solutions of the Degasperis-Procesi equation, Inverse Problems 19 (6) (2003) 1241-1245.

[28] H. Lundmark and J. Szmigielski, Degasperis-Procesi peakons and the discrete cubic string, IMRP Int. Math. Res. Pap. (2) (2005) 53-116.

[29] H. Lundmark and J. Szmigielski, An inverse spectral problem related to the Geng-Xue two-component peakon equation, Memoirs of Amer. Math. Soc. 244 (1155) (2016). 
[30] H. Lundmark and J. Szmigielski, Dynamics of interlacing peakons (and shockpeakons) in the Geng-Xue equation, Journal of Integrable Systems 2 (2017) 1-65.

[31] J. Lenells, A variational approach to the stability of periodic peakons, J. Nonlinear Math. Phys. 11 (2004), 151-163.

[32] Z. Lin and Y. Liu, Stability of peakons for the Degasperis-Procesi equation, Comm. Pure Appl. Math. 62 (2009), 125-146.

[33] J. Moser, Finitely many mass points on the line under the influence of an exponential potential-an integrable system, Lecture Notes in Phys. (Springer, Berlin, 1975), pp. 467-497.

[34] J. Moser, Three integrable Hamiltonian systems connected with isospectral deformations. Adv. Math. 16 (1975) 197-220.

[35] V. Novikov, Generalizations of the Camassa-Holm equation, J. Phys. A 42 (2009) 342002.

[36] P. Olver and P. Rosenau. Tri-Hamiltonian duality between solitons and solitary-wave solutions having compact support, Phys. Rev. E 53 (2)(1996) 1900-1906.

[37] Z. Qiao, A new integrable equation with cuspons and W/M-shape-peaks solitons, J. Math. Phys. 47 (2006) 112701.

[38] Z. Qiao and B. Xia, Integrable peakon systems with weak kink and kink-peakon interactional solutions, Front. Math. China 8 (5) (2013) 1185-1196.

[39] E. Recio and S.C. Anco, A general family of multi-peakon equations, arXiv: 1609.04354 math-ph

[40] J. Song, C. Qu and Z. Qiao, A new integrable two-component system with cubic nonlinearity, J. Math. Phys. 52 (1) (2011) 013503.

[41] T. Stieltjes, Recherches sur les fractions continues. OEuvres complètes de Thomas Jan Stieltjes, Vol. II (Nordhoff, Groningen, 1918), pp. 402-566.

[42] B. Xia, R. Zhou and Z. Qiao, A three-component Camassa-Holm system with cubic nonlinearity and peakons, J. Nonlinear Math. Phys. 22 (1) (2015) 155-169.

[43] B. Xia, Z. Qiao and R. Zhou, A Synthetical Two-Component Model with Peakon Solutions, Stud. Appl. Math. 135 (3) (2013) 248-276.

[44] B. Xia and Z. Qiao, Multi-component generalization of the Camassa-Holm equation, J. Geom. Phys. 107 (2016) 35-44.

[45] B. Xia and Z. Qiao, A new two-component integrable system with peakon solutions, Proc. R. Soc. A 471 (2015) 20140750.

[46] K. Yan, Z. Qiao and Z. Yin, Qualitative Analysis for a New Integrable Two-Component Camassa-Holm System with Peakon and Weak Kink Solutions, Commun. Math. Phys. 336 (2) (2015) 581-617. 